\title{
DELIMITACIÓN Y JUSTIFICACIÓN DE PROBLEMAS DE INVESTIGACIÓN EN CIENCIAS SOCIALES
}

\section{DELIMITATION AND JUSTIFICATION ON SOCIAL SCIENCES RESEARCH QUESTIONS}

\section{Diego Chaverri Chaves*}

RESUMEN

El inicio de un diseño de investigación social suscita varias interrogantes sustantivas; este artículo se dirige a dos de estas, las cuales consisten en problematizar sobre lo que es oportuno tomar en consideración cuando se delimita empíricamente una propuesta de investigación y su justificación, en tanto se fundamenta la elección de un tema; ambas cuestiones entrelazadas.

PALABRAS CLAVE: INVESTIGACIÓN * CIENCIAS SOCIALES * DISEÑO DE PROYECTO * ORGANIZACIÓN DE LA INVESTIGACIÓN * PROBLEMA SOCIAL

\section{ABSTRACT}

The starting point of a social research design brings up several substantial questions; this paper is addressed to two of them, which consist of problematizing on the considerations when engaging the empirical delimitation of the research proposal and its justification, namely, the choice of such a topic is grounded; both questions are inherently intertwined.

KEYWORDS: RESEARCH * SOCIAL SCIENCES * PROYECT DESIGN * ORGANIZATION OF THE RESEARCH * SOCIAL PROBLEM 


\section{JUSTIFICAR LO JUSTIFICABLE}

El primer paso del método científico es siempre un terreno movedizo, supone una primera decisión sobre la relevancia de un tema, la justificación es el espacio oportuno para dar argumentación convincente a tal selección, lo cual se puede lograr por varias vías. Aun cuando las motivaciones personales pesen de manera más o menos decisiva, sería ingenuo presumir que las preocupaciones individuales no tienen arraigo social, es decir, se trata de fundamentar la inquietud por conocer un aspecto particular de la realidad social más allá de un capricho intelectual, dándole objetividad a un paso ciertamente subjetivo, en tanto las concepciones de individuos científicos están condicionadas por factores históricos y sociales (Horkheimer, 2000).

Elegir el tema ya supone una delimitación. Por un lado, en muchas ocasiones, se trata de un "problema social", tal como lo define Berger (1992), esto sucede cuando una cultura tiene ciertas expectativas de cómo se debe comportar la sociedad y cuando estas no se cumplen, se diagnostica un problema, este último puede vulnerar a ciertos sectores de la población, por ejemplo, se espera que la infancia no padezca necesidades graves, de ahí que la hambruna infantil y la mortalidad asociadas a tal carencia, se consideran con toda razón un problema, empero, otros asuntos son mucho más vulnerables a estas predisposiciones culturales, así un divorcio puede ser considerado en algunos lugares o época como un problema, pero bajo otros parámetros de la vida doméstica, el divorcio puede ser entendido como otra alternativa más para la vida conyugal.

Esta primera tarea puede implicar algunas incomodidades, especialmente en el área de ciencias sociales, debido a que con frecuencia se lidia con justificar trabajos de investigación sobre aspectos referentes a la injusticia social, esto puede ejercer presiones de al menos dos tipos, primero, en orientaciones conservadoras se buscará la manera de maquillar el problema mediante eufemismos en el texto o del todo ignorar el alcance de ciertas formas de opresión y marginación social; segundo, en abordajes radicalizados el trabajo podrá tomar una vocación de denuncia social, con extremos incluso viscerales, es decir, de repudio hacia sectores de la población o individuos específicos.

En ambos casos se pierde la preocupación por plantear un problema de investigación, lo cual es un fallo recurrente, ya que no se interroga científicamente lo social, sino que se predica al respecto de las necesidades que le apremian. La alternativa es mantener un abordaje crítico, es decir, con cabida para la denuncia, pero interrogando cómo operan o se manifiestan los sectores dominantes de la sociedad, cómo se perpetúa o modifica la explotación, cómo resisten o sobreviven las personas marginadas, o cuáles son las consecuencias de ciertas formas de comportamiento, entre otras tantas preguntas, para así tener un problema de investigación que resolver, evitando el reduccionismo de lo social a su dimensión ética.

El conflicto social no es patrimonio exclusivo de las ciencias sociales, uno de los desafíos de estas es justamente el abordaje de temas sensibles para muchas otras personas. Las ciencias sociales no son solución inmediata de los problemas, algo así como la pócima última para la "sanación" de la sociedad, se debe proceder con modestia sobre los beneficios de la investigación, su impacto es parcial y tentativo, ineludiblemente afectado por su contexto de recepción (Aron, 1989).

El conocimiento que se logre puede favorecer el acompañamiento de procesos de transformación social, en tanto sea un saber público; las ciencias no redimen a la sociedad de sus problemas, pero sí pueden colaborar sensiblemente en discernir los aspectos que le aquejan; en el fondo, si se entiende cómo los conflictos son sociales, también se podrá entender que las alternativas son colectivas, no inventos individuales ni elaboraciones de un demiurgocientífico social.

El conocimiento obtenido de una ciencia social no supone imponerse a otros saberes, esto deriva muy fácilmente en proyectos autoritarios, pero sí puede presionar críticamente, aun cuando sea a través de la modesta labor científica. Se debe recordar que el discurso es también una forma de acción social y como tal 
puede tener efectos varios, de manera radical se debe renunciar a toda forma de ciencia conservadora, así como evitar una ciencia reducida a un proyecto individual de denuncia social sin salida. Preocuparse por la cientificidad de las disciplinas sociales no es un obstáculo para realizar otros aportes de tipo más práctico y transformador, solo desde una concepción ingenuamente conservadora de la ciencia se podría presumir que una mejor ciencia es totalmente ajena a problemas sociales, esto supondría ignorar que el contexto, es decir, las circunstancias históricas, culturales, políticas y económicas inciden de manera decisiva, tanto en las ciencias naturales como en las ciencias sociales, por eso los abordajes científicos de estos problemas son crecientemente intrincados (Elias, 2002).

De manera sucinta, la transformación de las relaciones sociales no es responsabilidad exclusiva del gremio científico, el cual también está sujeto a sus condiciones históricas y sociales, por tanto su involucramiento político no solo se manifiesta como una posibilidad, sino también en ocasiones como una necesidad, pues la ciencia no está destinada a reproducir un orden social, por el contrario, podría generar comprensiones capaces de superar la alienación social (Adorno, 2008).

Ahora, al problema científico social se le debe diferenciar del "problema social" antes mencionado, debido a que en el primer caso se entiende problema como tarea de investigación, se interroga lo social para averiguar por qué se comporta de tal manera, mientras que el problema social es una denuncia sobre las formas en que la sociedad discrepa con las expectativas o aspiraciones colectivas (Berger, 1992), nótese que el concepto mismo de "problema" es distinto, ya sea para asociarlo con carencia o conflicto, o con un emprendimiento investigativo, allí se interroga el problema social para conocer sus antecedentes históricos del problema, los factores novedosos o persistentes en el mismo, sus rasgos, sus principales actores y su comportamiento; todas estas preguntas pueden adquirir profundidad si hay un respaldo teórico, pues la teoría es sumamente enriquecedora para cuestionar y no solo como un acervo de respuestas.
El problema científico en una propuesta de investigación aún no producirá afirmaciones resolutivas sobre el problema, sino que lo interrogará, es allí donde la "justificación" en un diseño de investigación se encarga de señalar cómo dicho trabajo, a través de sus preguntas, podría llegar a ofrecer nuevos discernimientos, actualizar los datos disponibles, o buscar nuevas alternativas técnicas o metodológicas, entre otras opciones. Es decir, la justificación no está completa si se argumenta solo a favor de la relevancia social, también se debe resaltar la investigación misma, es decir, cómo aporta al estado de conocimiento de la disciplina sobre el tema elegido.

A pesar de las diferencias, no existe un antagonismo extremo ni inconciliable entre problemas sociales y problemas científicos; la justificación no puede depender exclusivamente de la gravedad del malestar social, pero tampoco puede ignorar sus contradicciones. La perspectiva crítica de la investigación, por ejemplo, puede investigar sobre conflictos o daños sociales, también puede interrogar aquellas relaciones que se presumen falsamente armónicas, pues la sociedad es de hecho contradictoria (Adorno, 2008), incluso se puede indagar sobre avances o novedades en formas de organización social con efectos beneficiosos para sus integrantes, lo cual significa que no todo problema científico social es reconocido como un problema social, aun cuando sea más fácil o urgente proceder a la inversa e identificar problemas científicos en problemas sociales, para poder restar ignorancia a aquellos aspectos de la vida social que nos generan disconformidad, daño físico o emocional, o incluso muerte.

En síntesis, la justificación puede señalar con la mayor brevedad posible cómo el diseño atiende un problema tanto social como científico de manera viable, esto quiere decir que está al alcance de los recursos de quienes investigan. Además, puede incluir los alcances tentativos que hacen estimable el trabajo, ya sea por beneficios a terceras personas derivados de la nueva sistematización de conocimiento que se podría brindar, por las innovaciones metodológicas, por la actualización de información, por 
el análisis de datos, por la exploración en un nuevo tema, entre otras.

En consecuencia, los potenciales aportes científicos pueden ser empíricos, metodológicos o teóricos; los aportes sociales pueden ser culturales, políticos, económicos, entre otras opciones, siempre de manera paradójica, pues solo con una preconcepción funcionalista conservadora de la sociedad se presume que la ciencia es siempre "buena" y quedará por resolver en procesos de más largo tiempo otras interrogantes como ¿Para qué sirve investigar este asunto? ¿Quién se beneficia de tal conocimiento? ¿Alguien se podría perjudicar con tal estudio? Sin embargo, la justificación señala cómo la investigación estará atenta sobre estos asuntos. En la investigación no se justifica un tema por su ausencia de dilemas éticos o porque se los resuelva, sino porque estos son incorporados como parte de la problematización científica (mucho de esto también atañe a las ciencias de la naturaleza).

\section{ELABORAR UNA IGNORANCIA CONOCIDA}

Una investigación no parte del supuesto de lo desconocido llanamente, las ciencias sociales tienen ya décadas de sumar avances empíricos en multiplicidad de temas, por tanto, cuando se señala la urgencia de una investigación por el vacío de conocimiento, usualmente es más por falta de insistencia en la localización de fuentes de información. El otro error es suponer un tema ya agotado, clausurar la investigación mediante una cabal búsqueda de información que incluso resuelve los mismos objetivos o preguntas de investigación, es decir, cuando prematuramente el estado de la cuestión no se orienta para discernir aspectos puntuales de la realidad que aún no han sido interrogados, sino solo para disipar las interrogantes con información de segunda fuente.

Ambos extremos deben evitarse en una investigación; es decir, considerar que hay un conocimiento total o nulo del tema; por el contrario, se trata de señalar mediante los avances de estudios previos cómo se puede identificar con cierta precisión uno o varios rasgos del tema, es decir, la mayor exhaustividad en la búsqueda de información pertinente y de calidad es útil para trazar continuidades, estimular el diálogo y contrastar entre distintos hallazgos, ya sea porque hay coincidencias entre datos de distinta fuente, lo cual permitiría avanzar hacia otros aspectos de la investigación, o cuando hay discrepancias entre los resultados, con lo cual se podría inquirir sobre las causas de tales fisuras en las conclusiones.

Se debe prestar atención a cómo los trabajos pueden divergir o converger según sus aproximaciones teóricas, sus propuestas metodológicas o por sus hallazgos empíricos. La novedad de la investigación que se diseña debe tener alguna conexión lógica con estos aspectos y ofrecer nuevos abordajes teóricos o metodológicos, asimismo, puede plantear hipótesis sobre el comportamiento empírico del fenómeno en cuestión.

En síntesis, esto es lo fundamental de los antecedentes (o "estado de la cuestión"), confeccionar una "ignorancia conocida", orientar la búsqueda de información para respaldar la relevancia del trabajo que se propone realizar, localizar y sustentar las preguntas de investigación como parte de un avance en campos del conocimiento. Aun cuando la propuesta sea exploratoria o abarque un tema nuevo, no se puede presumir nunca que nada en la investigación tiene parangón o antecedente, una vez más, la modestia investigativa se acompaña de un trabajo riguroso, los antecedentes de investigación ofrecen la oportunidad de acercar el diseño al diálogo con otros avances del campo científico. Es un recordatorio de que el avance en las ciencias es un esfuerzo colectivo y no una colección sucesiva de descubrimientos individuales.

De lo anterior se puede inferir la necesidad en la calidad de las fuentes, es decir, no se suele adquirir la misma calidad de información en una noticia de periódico en contraste con un artículo de revista científica que posee revisión de pares y otros rigores. En la medida de lo posible se deben rastrear las fuentes primeras, es decir, si se tiene una sinopsis sobre una base de datos pero también se tiene acceso a esa misma base de datos, es preferible atender con mayor cuidado la última. Otra razón por la cual muchas noticias no son la mejor fuente de información, es porque se encargan más de 
divulgar ciertos aspectos, que de interrogarlos o exponerlos con precisión, aunque caben excepciones ya sea por la calidad de un reportaje o por la investigación misma que se proponga hacer de las noticias un objeto de investigación.

Posteriormente, sin propósitos de censura alguna, la revisión documental también debe desarrollar herramientas para cernir la información obtenida en la web. Se recomienda evitar sitios del dominio ".com", lo cual significa que se trata de páginas con orientación comercial, por lo tanto, no sería una fuente académica, educativa, o científica. Asimismo, se deben evitar fuentes que no estén respaldas por una institución o autoría de una persona física, pues esto quiere decir que se publica anónimamente $y$ nadie respalda tal información, lo cual le resta fiabilidad, suelen ser fuentes de sentido común o meramente doxográficas (blogs o sitios de comentarios). Incluso, se hallan trabajos con problemas de plagios, no sistemáticos, sin una indagación seria.

Internet es una fuente muy amplia, da aforo también para informaciones erróneas, a las que en ocasiones es necesario oponer las fuentes físicas y desplazarse al lugar de origen de la información, o contrastar con el idioma original de la fuente citada, cuando es el caso que no se cuente con traducciones apropiadas.

En ciencias se entabla un fuerte empeño durante la revisión bibliográfica, sin embargo no todas las fuentes son escritas, se debe considerar la información disponible por otras vías de documentación, como los recursos audiovisuales; existen también otras fuentes de información, menos recurridas, pues suelen estar asociadas a temas de investigación muy específicos, como los museos, pinacotecas, hemerotecas, gliptotecas, entre otras (Gallardo, 2008).

Adicionalmente, no toda la información recabada tiene el mismo propósito, las fuentes se diferencian según la cantidad de información, el nivel de detalle de los tipos de datos disponibles, la pertinencia o cercanía con el tema, la vigencia o caducidad de la información; también puede variar el tipo de fuente, ya sea información testimonial (la palabra de agentes sociales individuales) o también institucionales (documentos o estadísticas oficiales de una empresa u organización). Los datos históricos o también la sistematización de experiencias metodológicas durante el trabajo de campo, son útiles en investigaciones que deben hacer inmersión en dinámicas de grupos con cierto nivel de hermetismo o suponen algún riesgo para quien investiga.

Para sintetizar, se pueden organizar los antecedentes de manera sencilla en tres tipos:

1. Los antecedentes empíricos ponen énfasis a toda la información disponible sobre las unidades de análisis para el trabajo de campo, por ejemplo, si se trata de personas se puede investigar de sus cualidades demográficas o las situaciones que atraviesan; en el caso de instituciones, se podrán localizar datos generales de estas (funciones, recursos u organización interna). Esta información también puede refrendar la viabilidad de la investigación, al prever si las fuentes serán receptivas de brindar información al ser interrogadas sistemáticamente, $y$ si posibilitarán el acceso a sus archivos o miembros. Toda esta información es aquella que se puede obtener aún sin aplicar técnicas de investigación más allá de la consulta de documentos o sondeos no sistemáticos. Es importante considerar un diseño juicioso en la investigación desde la dimensión documental, ya que será el cimiento en la forma final que tomará el diseño de investigación.

2. Los antecedentes históricos, también son accesibles de manera documental. Se pueden incluir reseñas cronológicas de un acontecimiento, la trayectoria biográfica de algún informante clave o los orígenes de una institución. Esto supone elaborar también algo de la dimensión histórica de lo social, inscribir los hechos que se quieren indagar en una trama de sentido histórico, para poder así desentrañar cómo ha venido transformándose el problema de investigación, si han aparecido nuevas tendencias, rasgos singulares propios de un contexto cultural, o propios de una orientación dominante en 
una corriente mucho más global. No se trata de elaborar todo lo que antecede en el tiempo a un problema social, sino los principales hitos de la coyuntura histórica en que se inscribe el tema de investigación.

3. Los antecedentes investigativos se orientan a ponerse al corriente sobre la manera en que el tema se ha venido investigando, el predominio de un cierto tipo de técnica o la combinación de varias metodologías, la realización recurrente de ciertas preguntas de investigación, la discontinuidad de algún aspecto de la investigación o el surgimiento de nuevas interrogantes. Todos estos antecedentes de tipo metodológico pueden ofrecer alguna previsión de las dinámicas que se presentarán en el trabajo de campo, dar ideas para replantear el tema o las preguntas de investigación, analizar diversas alternativas para el diseño de instrumentos y la planificación de la logística del trabajo de campo. De esta manera se podrá conectar directamente la congruencia del trabajo desde la consulta de antecedentes hasta la propuesta metodológica.

Finalmente, la sencillez de esta tipología de los antecedentes tiene algo de ficticio, los documentos hallados pueden mezclar mucha de esta información, por lo cual la pericia del investigador o investigadora definirá cómo organizar sus antecedentes; no se trata tanto de la forma, sino que el contenido de esta sección no descuide tales tipos de fuentes y se procure equilibrar la búsqueda de investigación según los aspectos más importantes para confeccionar justamente la ignorancia conocida del problema de investigación.

\section{DELIMITACIÓN Y ARTICULACIÓN}

La justificación del problema de investigación y la delimitación se enlazan con un aspecto importante con el que se debe guardar coherencia: el título. El nombre general de una propuesta surge no solo de la revisión de fuentes, también de la creatividad y capacidad explicativa de quien investiga, así como de un ejercicio de delimitaciones, ya que el nombre de la obra puede permitir incluso advertir límites de la investigación, a saber la delimitación espacial, en la cual se detalla el lugar, ya se trate de una investigación global, regional, nacional o local, incluso circunscrita a espacios institucionales y por otro lado, la delimitación temporal, la cual señala la extensión de tiempo que cubre el estudio, sea de meses o incluso años, de manera constante $\mathrm{u}$ observando intervalos de tiempo. Lo que se busca siempre es indicar con claridad el contexto geográfico e histórico en el cual queda circunscrito el trabajo de campo realizado en el proyecto de investigación.

Otra forma de establecer fronteras empíricas se logra con la delimitación poblacional, según el caso, cuando se trabaja con personas informantes, se debe precisar su perfil demográfico, es decir, a las características referidas al sexo, edad, etnia, estrato ocupacional, situación económica, nivel de escolaridad, zona de residencia, nacionalidad, entre otras muchas cualidades básicas, según se combinen para el problema abordado, así se logra precisar las condiciones desde las cuáles las personas consultadas ofrecen testimonio de su experiencia sobre el tema indagado. Esto también se puede aplicar cuando se trabaja con organizaciones, en especial cuando tienen varias sedes o sucursales, es importante localizarlas e indicar en cuáles se trabajará. Existen temas en los cuales la referencia poblacional e institucional puede coincidir, esto cuando en el trabajo de campo se localiza informantes relevantes para el estudio.

Enfocar el trabajo no supone acortarlo, puede ofrecer simultáneamente la opción de profundizar en algunos escenarios o sobre ciertos agentes, no obstante, es importante incluir una delimitación conceptual. Pueden existir diversos criterios teóricos al inicio de una investigación, en aspectos como el tema, una población, un lugar, un momento; no obstante, falta indicar el aspecto de lo social que se indagará, ya que la delimitación conceptual evitará querer interrogarlo todo a nivel empírico sobre el contexto predefinido. Se plantea el problema en sentido teórico, es decir, toda delimitación requiere ser simultáneamente una articulación con su contexto (Zemelman, 1992), todas las 
señas limítrofes de una propuesta de investigación permiten diseñar un trabajo de campo mucho más preciso respecto de su indagación empírica y a la vez, permiten reflejar orientaciones sobre los contenidos del documento y cómo se cuestionan sus relaciones con otros aspectos de lo social.

El esfuerzo de articulación se encarga además de procurar congruencia entre los distintos segmentos de una propuesta de investigación (algunos que por extensión no se consideran en este documento, como la metodología o la teoría), y puede verse expresado en la transparencia entre los objetivos y la pregunta de investigación, tanto en su redacción como en su formulación lógica. La pregunta de investigación recoge todas las delimitaciones anteriores y las une en la forma de la interrogante central del trabajo, esto posibilita que, existiendo múltiples facetas de lo social, el diseño tenga claridad respecto de su preocupación central, logrando una jerarquía de problemas. Por lo tanto, la pregunta de investigación logra señalar ese aspecto relacional a partir del cual se problematizará el tema elegido; no se trata de elucubrar erotemas, sino de forjar preguntas que puedan responderse mediante contrastación empírica y sustento teórico en el marco de una investigación.

Por su parte, el objetivo prosigue la delimitación y reformula la pregunta de investigación en la forma del propósito central del estudio, es decir, aquello que se quiere responder apelando al trabajo de campo, con su posterior sistematización y exposición de hallazgos. Sobre la distinción entre objetivo general y objetivos específicos, lo fundamental está en lograr desagregar lo general, es decir, cómo se puede subdividir la tarea propuesta en el objetivo general, de modo tal que los objetivos específicos logren una secuencia, se mantengan coherentes e insistan en cómo se entiende el carácter relacional de lo social en el diseño.

Para ilustrar un poco lo anterior, sirva de muestra que un objetivo general dado plantea que se quiere comprender qué relación existe entre un "X" que se comporta de manera "A" en un contexto " $Z$ ", luego los objetivos específicos podrán precisar que para lograr tal explicación, primero se deben describir los rasgos principales de "X", "A" y "Z", otro objetivo servirá para señalar los vínculos entre "X", "A" y "Z", y un objetivo más plantea la indagación respecto de la relación entre los rasgos y los vínculos de "Z", "A" y "X". Esto no es una fórmula, solo un ejemplo, cada investigación atenderá distintos tipos de problema, lo verdaderamente importante de este señalamiento es entender cómo el objetivo general se puede desagregar en distintas facetas en los objetivos específicos, de tal manera:

\section{OBJETIVO GENERAL}

Comprender la relación que existe entre el comportamiento "A" de un "X" en el contexto "Z".

\section{OBJETIVOS ESPECÍFICOS}

Describir los principales rasgos de "X", "A" y "Z".

Definir los principales vínculos entre "X", "A" y "Z".

Explicar la relación entre los rasgos y los vínculos de "X", "A" y "Z".

Se puede notar cómo estas observaciones discuten la congruencia del planteamiento, no su mera diagramación. Otra advertencia provechosa para la redacción de objetivos, es mantener la congruencia con la delimitación conceptual, ya que introducir un concepto en el objetivo general y luego plantear un sinónimo de uso coloquial, puede significar distorsiones en la rigurosidad del problema; un breve ejemplo de esto, el objetivo general apunta a conocer sobre la "marginación", pero luego los conceptos de los objetivos específicos señalan otros asuntos, como la "expulsión", la "opresión”, y la "dominación”, aunque en su uso vernáculo o incluso retórico puedan tener definiciones equivalentes, en sentido investigativo tienen diferencias teóricas y metodológicas, por eso el parámetro para decidir los conceptos clave se desarrolla en enlace directo con los supuestos teóricos.

La ciencia requiere ciertamente un momento de descripción e incluso de retrodicción (Fernández, 1992), luego avanza mediante la 
insistencia hacia la explicación de los principales factores que inciden en un fenómeno y la comprensión de la singularidad del caso estudiado. La pregunta y los objetivos de investigación deben resolver de la manera más concisa posible a quiénes se investiga, para averiguar qué, cómo, dónde y cuándo, logrando así articular el proceso de diseño con todos los segmentos del trabajo. El diseño de una propuesta de investigación siempre es preliminar, la redacción de objetivos y preguntas podrá pasar por varias formulaciones hasta lograr articular los restantes segmentos de la propuesta de investigación.

\section{POR ÚLTIMO: LA INTRODUCCIÓN}

La introducción es siempre la sección inicial del documento de diseño, pero la sugerencia no es cambiarla de orden, sino simplemente que sea la última parte redactada del diseño. $\mathrm{Su}$ contenido es variable, pues puede elaborar argumentos alrededor de la idea de persuadir o disuadir potenciales lectores, sugerir la utilidad de la investigación o la relevancia del tema, pero muy frecuentemente la introducción ofrece un resumen de contenidos para saber qué se encuentra en el texto y cuál es su secuencia. Para esta fase, es útil considerar uno o varios índices, el principal y más recurrente es el índice de contenidos, pero también puede aparecer un índice de gráficos o imágenes, en investigaciones muy especializadas hasta se puede elaborar un índice onomástico o analítico.

En este punto es relevante considerar el proceso de escribir la investigación una vez culminado el trabajo de campo e indagación de fuentes. Se dan importantes diferencias en los escritos de una investigación, una de estas es que mientras el informe final de un proyecto trabaja con errores, el anteproyecto se trabaja predominantemente con la previsión de errores; no obstante, escribir en ciencias sociales supone descubrir y crear, hay un sentido de poiesis (Bauman, 2003), en la cual el esfuerzo se orienta por buscar la mejor manera de exponer los resultados.

La redacción que se logre, no solo en el segmento de "introducción", sino a lo largo de toda la propuesta de investigación (e incluso, el informe de la investigación), ya sea aquella intrincada o sencilla, no debe perjudicar el trabajo de investigación, el propósito es lograr la mayor inteligibilidad posible, pero el lenguaje coloquial no siempre alcanza para esta meta, requiere que quienes se instruyan y lean sobre las disciplinas, se armen de la terminología necesaria, como en toda ciencia, para puntualizar sobre las dimensiones de lo real que se quiere conocer. Entonces, si lo social es complejo, el texto en consonancia tendrá también algún nivel de complejidad, lo demás sería suponer que lo social es inmediata y fácilmente comunicable en la escritura, $y$ por extensión que el lenguaje es totalmente diáfano; ni una ni otra, el lenguaje también resulta a la vez socialmente posibilitado y determinado (Lefebvre, 1967). Ahora bien, si lo que se plantea es un problema político del alcance del conocimiento a otras poblaciones, entonces se debe trabajar en la educación de tales personas y la capacidad educativa de quienes trabajan en ciencias, para nutrirlas del conocimiento necesario que les faculte para ser entendidas en la materia, poder compartir sus conocimientos y producir nuevos saberes, pero nunca engañar a las audiencias con versiones procesadas, remedos de textos científicos cuyo vacío se debe a la simplificación en exceso; de igual manera se debe censurar la presuntuosidad de cualquier científico que renuncie a sus responsabilidades educativas.

La ciencia forzosamente tiene que ser comunicativa pero sin traicionar la densidad explicativa que requiere un objeto de investigación científico, esto es lo que finalmente prevalece, pues un texto adornado estilísticamente o lexicalmente ampuloso, puede perfectamente ser torpe en términos científicos y prestarse para infatuaciones por vía de la criptolalia (Andreski, 1973), o aprovecharse de la combinación de terminologías (en ocasiones procedentes de varias disciplinas) sin detenerse primero a cimentar la articulación antes mencionada entre los conceptos y las preguntas empíricamente delimitadas. Además, escribir en ciencias sociales requiere tomar distancia y tiempo (Bauman, 2003), es decir, por una parte es un proceso de extrañamiento para no forzar la discusión desde la mera familiaridad, cayendo en lugares 
comunes, $y$ por otra parte, requiere reflexión detallada, interiorizar el problema para poder hacerlo comunicable.

La ciencia social cuando es crítica, es incómoda, armada de un aparato teórico y empírico, con un discurso responsable, dispuesto al debate público, a la confrontación de hallazgos; esto requiere profundizar cada cierto tiempo en la exposición de resultados, ninguna tesis verdaderamente científica se puede quedar restringida a un nivel meramente descriptivo, esto no brinda las respuestas, aún faltaría indicar cuáles son las razones sociales para que el fenómeno estudiado tenga tales cualidades y no otras. Sobre la crítica, no es solo una opción política, puede resultar también una necesidad, una sociedad enferma es la que no se cuestiona, pero desdichadamente conocer no basta para remediar (Bauman, 1994), este es otro llamado a la modestia científica sin abandonar sus ambiciones.

La ciencia es un proyecto que entre otras cosas plantea la apertura del conocimiento, esto puede generar disconformidad en ciertos sectores de la sociedad conservadores 0 favorecidos por el mantenimiento de la ignorancia cultural, por eso superar una condición de malestar social quizá pueda llegar a suscitar molestias.

Entonces las ciencias sociales pueden interrogar sobre el malestar y sus respuestas pueden llegar a molestar; reconocer los propios defectos para cualquier grupo humano, no es tarea sencilla, la utilidad de las ciencias sociales puede resultar eventualmente paradójica, sino ambivalente; no es inusual que una persona investigadora, al reconocer la complejidad de un tema se atemorice y prefiera cambiar de asunto, esto es inconsecuente con lo expuesto en este documento. No hay temas fáciles, solo quizá modos simplificados o escuetos de abordaje, lo social no es un asunto llano, al menos no desde una perspectiva crítica, y esta se logra trabajando con cuidado en los detalles de inicio de una investigación, desde su justificación y su delimitación, con el propósito de hacer mejor ciencia con mayor impacto.

\section{REFERENCIAS}

Adorno, T. (2008). Introducción a la sociología. España: Editorial Gedisa.

Andreski, S. (1973). Las ciencias sociales como forma de brujería. España: Editorial Taurus.

Aron, R. (1989). Estudios sociológicos. España: Editorial Espasa-Calpe.

Bauman, Z. (1994). Pensando sociológicamente. Argentina: Ediciones Nueva Visión.

Bauman, Z. (2003). Modernidad líquida. México: Fondo de cultura Económica.

Berger, P. (1992). Introducción a la sociología. México: Editorial Limusa.

Elias, N. (2002). Compromiso y distanciamiento. España: Ediciones Península.

Fernández, Oscar (1992). Sobre predicciones y relatos. Revista de Filosofía, 71 . Universidad de Costa Rica, Costa Rica.

Gallardo, H. (2008). Fundamentos de Investigación Académica. Costa Rica: Editorial Universidad Estatal a Distancia.

Horkheimer, M. (2000). Teoría tradicional y teoría crítica. España: Ediciones Paidós Ibérica.

Lefebvre, H. (1967). Lenguaje y Sociedad. Argentina: Editorial Proteo.

Zemelman, H. (1992). Los horizontes de la razón: uso crítico de la teoría. México: Editorial Anthropos.

Fecha de ingreso: 19/08/2016 Fecha de aprobación: 11/07/2017 
